University of Pittsburgh

Department of Electrical Engineering

Pittsburgh, Pennsylvania

\title{
ON THE STABILTYY OF NONLONEAR OPERATOR
}

DIFPERENTUAL EOUATUONS, AND APPLUCATUONS

\section{BY}

C. V. PAO

WILLIAM G. VOGT

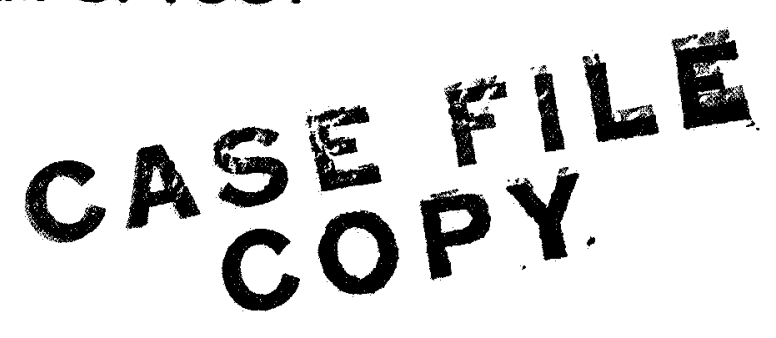

\author{
prepared for the \\ NATIONAL AERONAUTICS AND SPACE ADMINISTRATION \\ under
}

Grant Number NGR 39-011-039 


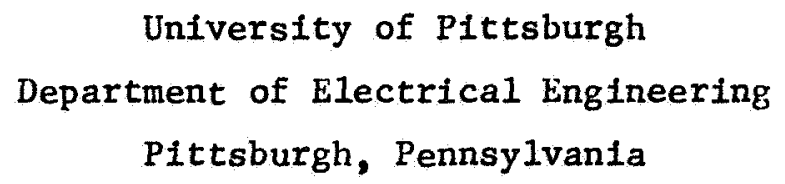

ON THE STABILITY OF NONLINEAR OPERATOR DIFFERENTIAL

$$
\text { EQUATIONS, AND APPLICATIONS }
$$

prepared by

C. V. Pao

Scientific Investigator
William G. Vogt

Princlpal Investigator

$$
\text { for the }
$$

National Aeronautics and Space Administration

under

Grant Number NGR 39-011-039

Apri1 27, 1969 
NOTICE

The research reported herein was partially supported by the National Aeronautics and Space Administration under Grant Number NGR 39-011-039 with the Universfty of Pittsburgh. Reproduction in whole or in part is permitted for any purposes of the United States Government. Neither the National Aeronautics and Space Administration nor the University of Pittsburgh assumes responsibility for possible inaccuracies in the content of this report. 
ON THE STABILITY OF NONLINFAR OPERATOR DIFFERENTIAL

$$
\text { EQUATIONS, AND APPLICATIONS }
$$

by

$$
\text { C. V. Pao William G. Vogt }
$$

Department of Electrical Engineering

University of Pittsburgh

Pittsburgh, Pennsylvania

\section{ABSTRACT}

Consider the nonlinear operator differential equation

(1.e., equation of evolution)

$$
\frac{d x(t)}{d t}=A x(t)+f(x(t)) \quad(t \geq 0)
$$

where $A$ is a linear (unbounded) operator wtth domain and range both in a real Hilbert space $H$ and $f$ is a (nonlinear) function defined on $\mathrm{H}$ into $\mathrm{H}$. The object of this paper is to investigate the existence, the uniqueness and the stabllity or asymptotic stability of solutions to (*) by using nonlinear semi-group properties. Criteria on $\mathrm{A}$ and on $\mathrm{f}$ for the generation of a contraction or negative contraction semi-group are established from which the existence, uniqueness, stability and asymptotic stabllity of solutions of (*) are Insured. Applications are given to the second order partial differential equation of the form

$$
\frac{\partial u}{\partial t}=\sum_{1, j=1}^{n} \frac{\partial}{\partial x_{i}}\left(a_{i j}(x) \frac{\partial u}{\partial x}\right)+c(x) u+f(x, u) \quad x \varepsilon \Omega<R^{n} .
$$

Criteria in terms of the coefficients $a_{i j}(x), c(x)$ and of the function f are obtained. 
ON THE STABILITY OF NONLINEAR OPERATOR DIFFFRENTIAL EQUATIONS, $\triangle N D$ APPLICATIONS

\section{Introduction}

This paper is concerned with the existence and the stability nroblems of the onerator differential equations (i.e., equations of evolution) of the form

$$
\frac{d x(t)}{d t}=A x(t)+f(x(t)) \quad t \geq 0
$$

where $A$ is a linear, in general unhounded, operator with domain $D(A)$ and range $R(A)$ both contained in a real Hilbert space $H$ and $f$ is a (nonlinear) function defined on $\mathrm{H}$ into $\mathrm{H}$. It is well known that some semi-1inear systems of differential equations, hoth ordinary and partial, can he reduced to the form (1-1) and in such cases A may be considered as an extension of a linear differential operator. In order to examine the stablitty of solutions to (1-1), it is onlv necessary to characterize their properties without actually constructing the solutions. This ts done be considering the properties of a nonlinear semi-groun because if the operator $A+f(\cdot)$ generates a nonlinear semigroun $\left\{T_{t} ; t \geqq 0\right\}$ (see definition 2.1) then a solution to (1-1) startinc at $t=0$ from anv element $x_{0} \varepsilon D(A)$ is given by $x\left(t ; x_{0}\right)=T_{t} x_{0}$ for a11 $t \geqq 0$ with $x\left(n ; x_{0}\right)=x_{0}$. Thus the existence of a solution to (1-1) is ensured and the stability property can be determined from the family of nonlinear operators $\left\{T_{t} ; t \geqq 0\right\}$. The object of this paper is to impose conditions on the operators $A$ and $f$ such that the operator $A_{1} \equiv A+f(\cdot)$ generates a nonlinear contraction or negative contraction semi-group in $H$ or in an equivalent Hilbert space of $H$ (see definition 2.4) from which the existence, uniqueness and stability or asymptotic stability of solutions to $(1-1)$ are insured. 
The following definitions specifies what we mean by a sclution, an equilthrium solution and the stability of an unerturhed solution.

Definition 1.1. Py a solution $x(t)$ of $(1-1)$ with inftial conditions $x(0)=x \in D(A)$ in a Hilhert space $H$, we mean the following:

(a) $x(t)$ is uniformly Lipschitz continuous in $t$ for each $t \geqq n$ with $x(0)=x$

(b) $x(t) \in D(A)$ for each $t \geqq n$ and $A x(t)+f(x(t))$ is weakly continuous in $t$;

(c) the weak derivative of $x(t)$ exists for all $t \geqslant n$ and equals $A x(t)+f(x(t))$;

(d) the strong derivative $\frac{d x(t)}{d t}(=A x(t)+f(x(t))$ exists and is strongly continuous excent at a countable number of values $t$.

The above definition of a solution $x(t)$ is in the sense of a weak solution since $x(t)$ satisfies (1-1) in the weak topology of $H$. However, by the condition $(d), x(t)$ is an almost everywhere strong solution in the sense that $x(t)$ satisfies $(1-1)$ for almost all values of $t \geqq 0$ in the strong tonology of $\mathrm{H}$.

Definition 1.2. An equilibrium solution of (1-1) is an element $x_{e}$ in $D(A)$ satisfying (1-1) (In the weak topology) such that for any solution $x(t)$ of $(1-1)$ with $x(n)=x_{e}$

$$
|| x(t)-x_{e}||=0 \quad \text { for all } t \geq 0 \text {. }
$$

It can be shown that if $x(t)$ is a solution of (1-1) then it is an equilibrium solution if and only if $A x(t)+f(x(t))=0$ for all $t \geqq 0$ (cf [9]).

Definition 1.3. An equilibrium solution (or any unperturbed solution) $x_{e}$ of (1-1) is said to he stable (with respect to initial perturbations) if given any $\varepsilon>0$, there exists a $\delta>0$ such that 


$$
\left\|x-x_{e}\right\|<\delta \text { Implies }\left\|x(t)-x_{e}\right\|<\varepsilon \quad \text { for all } t \geq 0 \text {; }
$$

$x_{e}$ is said to be asymptotically stable if

(1) It is stable; and

(1i) $\lim _{t \rightarrow \infty}\left\|x(t)-x_{e}\right\|=0$

where $x(t)$ is any solution of (1-1) with $x(0)=x \in D(A)$. If there exist positive constants $M$ and $B$ such

$$
\text { (ii)' } \| x(t)-x_{e}|| \leqq M e^{-\beta t}|| x-x_{e}|| \text { for all } t \geqq 0
$$

then $x_{e}$ is called exponentially asymptotically stable.

The existence problem for the case of a general nonlinear equation

of evolution

$$
\frac{d x(t)}{d t}=A_{1} x(t)
$$

where $A_{1}$ is a general nonlinear operator with $D\left(A_{1}\right)$ and $R\left(A_{1}\right)$ both In $\mathrm{H}$, has been Investigated by Kömura [6], Kato [5] and by Browder [2]; and the stability problem of the same type of equation has been studied by the author in a separate paper [9] which has a close connection with the nresent work. Because of the Implication of a general nonlinear operator differential equation, the application of its results to partial or ordinary differential equation needs additional justification. However, In the case of semi-linear equation of the form (1-1), criteria on the operator $A$ and on the function $f$ are directly applicable to certain partial or ordinary differential equations. Examples of application to partial differential equations are given in the last section in order to 1llustrate certain stens in applying the results developed for onerator differential equation. Further applications to nonlinear partial differential equations w111 be discussed in a separate presentation. 


\section{General Background}

In this section, we shall introduce some basic definitions and state some theorems from [9] which are fundamental in the develoment of our results.

Definition 2.1. Let $\mathrm{H}$ be a Hilbert snace. The family of operators $\left\{T_{t} ; t \geqslant 0\right\}$ is called a nonlinear semi-group on $H$ if and only if the following conditions hold:

(1) for any fixed $t \geq 0, T_{t}$ is a continuous (nonlinear) operator defined on $\mathrm{H}$ into $\mathrm{H}$;

(11) for any fixed $x \in H, T_{t} x$ is strongly continuous in $t$;

(iii) $T_{s} T_{t}=T_{s+t}$ for $s, t \geq n$, and $T_{0}=I$ (the identity operator); (iv) ||$T_{t} x-T t_{t} y|| M|| x-y||(M>0) x, y, \varepsilon H$ and $t \geqq 0$.

If (Iv) is replaced by

(Iv)' $\left\|T_{t} x-T_{t} y\right\| \leqq M e^{-\beta t}|| x-y \|(\beta>0) x, y \in H$ and $t \geqq 0$ then $\left\{T_{t} ; t \geq 0\right\}$ is called a (nonlinear) negative semi-group; if $M \leqq 1$ then it is called a (nonlinear) contraction or negative contraction semi-group according to (iv) or (iv)' respectively. The number $\beta$ satisfying (iv)' is called a contractive constant of $\left\{T_{t} ; t \geq 0\right\}$. For a subset $D$ of $H$, the family $\left\{\mathrm{T}_{t} ; t \geq 0\right\}$ is said to be a nonlinear contraction (resp., negative contraction) semi-groun on $D$ if the properties (1)-(iv) (resp.,(i)-(iv)') are satisfied with $M \leqq 1$ ) for all $x, y \in D$. Definition 2.2. The infinitesimal generator $A_{1}$ of the nonlinear semi-group $\left\{T_{t} ; t \geqq 0\right\}$ is defined by

$$
A_{1} x=\underset{h+0}{w} \frac{T_{h} x-x}{h}
$$

for all $x \in H$ such that the limit on the right-side exists in the sense of weak convergence. 
Definition 2.3. An operator (nonlinear) $A_{1}$ with domain $D\left(A_{1}\right)$ and range $R\left(A_{1}\right)$ both contained in a real Hilbert space is sald to be dissinative if

$$
\left(A_{1} x-A_{1} y, x-y\right) \leq 0 \quad \text { for } x, y \in D\left(A_{1}\right) \text {; }
$$

and $A_{1}$ is called strictly dissipative if there exists a real number $B>0$ such that

$$
\left(A_{1} x-A_{1} y, x-y\right) \leq-B|| x-y||^{2} \quad x, y \in D\left(A_{1}\right) \text {. }
$$

The supremum of all numbers $\beta$ satisfying $(2-2)$ is called the dissipative constant of $\mathrm{A}_{1}$.

It follows from the above definition that $A_{1}$ is dissipative if and only if $-A_{1}$ is monotone and $A_{1}$ is strictly dissipative if and only if there exists a real number $B>0$ such that $-\left(A_{1}+B I\right)$ is monotone (cf [8]). Note that definition 2.3 coincides with the usual definition of dissipativity when $A_{1}$ is a linear operator (cf. [7]).

Definition 2.4. Two inner products (.,.) and $(,,)_{1}$ defined on the same vector space $H$ are said to he enulvalent if and only if the norms $\|\cdot\|$ and $\|\cdot\|_{1}$ induced by $(\cdot, \cdot)$ and $(\cdot,)_{1}$ respectively are equivalent, that is, there exist constants $\delta, \gamma$ with $0<\delta \leqq \gamma<\infty$ such that

$$
\delta\|x\| \leqq\|x\|_{1} \leqq \gamma\|x\| \text { for all } x \in H \cdot(2-3)
$$

The Hilbert space $H_{1}$ equipped with the inner product $(\cdot, \cdot)_{1}$ is sald to be an equivalent $H i l b e r t$ space of $H$ and is denoted by $\left(H,(\cdot, \cdot)_{1}\right)$ or simply by $\mathrm{H}_{1}$.

In order to show the results in the following sectlons we state some tesults from [9].

Theorem 2.1. Let $A_{1}$ be a nonlinear operator with domain $D\left(A_{1}\right)$ and range $R\left(A_{1}\right)$ both contained in a Hilbert space $H$ such that $R\left(I-A_{1}\right)=H$. 
Then $A_{1}$ is the Infinitesimal generator of a nonlinear contraction semi-group on $D\left(A_{1}\right)$ if and only if $A_{1}$ is dissipative; and $A_{1}$ is the Infinitesimal generator of a nonlinear negative contraction semi-groun if and only if $A_{1}$ is strictly dissipative.

Theorem 2.2. If $A_{1}$ is the infinitesimal generator of a nonlinear contraction semi-group (resp., negative contraction semigroup) in an equivalent Hilbert space on $D\left(A_{1}\right)$ then $A_{1}$ is the infinitesimal generator of a nonlinear semi-group (resp., negative semi-group), not necessarily contractive, on the same domain $D\left(A_{1}\right)$ in the original Hilbert space.

Remarks: (a) In Theorem 2.1, the condition $R\left(I-A_{1}\right)=H$ can be weakened by $R\left(I-\alpha A_{1}\right)=H$ for some $\alpha>0$. (b) The nonlinear contraction semi-group $\left\{T_{t} ; t \geqq 0\right\}$ generated by $A_{1}$ in the above theorem has the following additional property: For any $x \in D\left(A_{1}\right)$, the strong derivative $\frac{d\left(T_{t} x\right)}{d t}=A_{1} T_{t} x$ exists and is strongly continuous except at a countable number of values $t$ (cf. [5]). Thus for any $x \in D\left(A_{1}\right), T_{t} x$ is a solution of (1-1) In the sense of definition 1.1 .

It is seen from theorem 2.1 that $i f$ the operator $A+f(\cdot)$ is dissipative or strictly dissipative and $R(I-A-f(\cdot))=H$ then $A+f(\cdot)$ is the infinitesimal generator of a contraction and negative contraction semi-group respectively. However, the requirement $R(I-A-f(\cdot))=H$ by itself is not easy to verify since it is equivalent to the functional equation

$$
x-A x-f(x)=z
$$

having a solution for every $z \varepsilon \mathrm{H}$. In the following section, we sha11 Impose conditions on $A$ and $f$ to insure the existence of a solution of $(2-4)$. We consider first the case that $A$ is the infinitesimal generator of a linear contraction (or negative contraction) semi-groun 
of class $C_{0}$ (cf. [11]), and then consider the more general case when $A$ is an unbounded closed operator. Notice that the infinitesimal renerator of a semi-group is always closed.

3. Existence and Stability of Solutions

In the proof of the main theorems in this section, we have used some results developed hy Browder (cf. [1], [2]). It is noted that a Hilbert space is reflexive and uniformly convex and the definition of an accretive operator defined in [2] coinctides with a monotone onerator when the underlying space is a pilbert snace.

Definition 3.1. Let $x(t)$ he a solution to $(1-1)$ with $x(n)=x$. A subset $D$ of $I$ is said to be a stability region of the equilihrium solution (or any unnerturbed solution) $\mathrm{x}_{\mathrm{e}}$ if for any $\varepsilon>0$ there exists a $\delta>0$ such that

$$
x \in D \text { and } \| x-x_{e} \mid<\delta \text { imp } 1 y \quad\left\|x(t)-x_{e}\right\|<\varepsilon \quad \text { for all } t \geq n \text {. }
$$

Theorem 3.1. Let A be the infinitesimal penerator of a (linear) contraction semi-group of class $C_{0}$. Assume that $f$ satisfies: (1) $f$ is defined on all of $I I$ into $I$ such that it is continuous from $H$ in the strong tonology to the weak tonology, and is hounded on every bounded subset of $H$. (ii) for any $x, y \in H,(f(x)-f(y), x-y) \leqq 0$. Then (a) for any $x \in D(A)$, there extsts a unique solution of (1-1) (in the sense of definition 1.1) with $T_{0} x=x$; (b) any equilibrium solution $x_{e}$ (or any unperturhed solution such as periodic solution), if it exists, is stable; (c) a stability region of $x_{e}$ is $D(A)$ which can be extended to $\mathrm{H}$.

Proof. Let $A_{1}=A+f(\cdot)$ with $D\left(A_{1}\right)=D(A)$. Since an infinitesimal generator of a contraction semi-group of class $C_{0}$ is 
densely defined, dissipative and $R(I-A)=I 1$ (cf. [7] or [11]), it follows by the dissipativity of $A$ and by the assumption (ii) on $f$ that

$$
\left(A_{1} x-A_{1} y, x-y\right)=(A x-A y, x-y)+(f(x)-f(y), x-y) \leqq 0 \text { for a11 } x, y \varepsilon D\left(A_{1}\right)
$$

which shows that $A_{1}$ is dissipative. To show that $R\left(I-A_{1}\right)=H$, we apply a theorem (theorem 5) in [2]. Tote that the onerator $-\Lambda$ is monotone and the range of $-A+I$ is all of $F$ with $D(-A)=D(A)$ dense in $\mathrm{H}$. Thus the operator $\mathrm{G}=-\mathrm{A}$ is accretive (or monotone). Let $G_{0}=I-f(\cdot)$, then from assumption $(i) G_{0}$ is defined on all of $H$ and is continuous from $H$ in the strong topology to the weak tonology (i.e., $G_{0}$ is demicontinuous on $F$ ) and mans hounded subsets of $H$ into bounded subsets of $H . G_{0}$ is monotone, for

$$
\left(G_{0} x-G_{0} y, x-y\right)=(x-y, x-y)-(f(x)-f(y), x-y) \geqq\|x-y\|^{2} x, y \varepsilon \text { II }
$$

where we have used assumption ( $1 i$ ). Moreover, by letting $y=0$ in (ii) gives

$$
(f(x), x) \leqq(f(0), x) \leqq \| f(0)|!||x| \mid \quad \text { for all } x \in H
$$

It follows by the dissipativity of $A$ and by (3-1) that

$$
\begin{aligned}
\left\|-A x+G_{0} x\right\| & \geqq\left(-A x+G_{0} x, x\right) /|| x\left\|\geqq\left(G_{0} x, x\right) /\right\| x\|=((x, x)-(f(x), x)) /\||x| \mid \\
& \geqq\|x\|-\|f(0)\| \text { for al1 } x \in D(A) \quad(x \neq 0) .
\end{aligned}
$$

Thus $\| G x+G_{0} x||++\infty$ as ||$x||+\infty$. Hence all the hypotheses in theorem 5 of $[2]$ are satisfied. It follows that $R\left(I-A_{1}\right)=R\left(G+G_{0}\right)=H$. This later condition and the dissipativity of $A_{1}$ imply that $A_{1}$ is the infinitesimal generator of a nonlinear contraction semi-group $\left\{T_{t} ; t \geqq n\right\}$ on $D(A)$ by applying theorem 2.1. Therefore, for any $x \in D(A), T_{t} x \in D(A)$ and is the unique solution of $1-1$ with $T_{0} x=x$. Since

$$
\| T_{t} x-T_{t} y|| \leqq|| x-y|| \text { for all } t \geqq 0 \quad x, y \in D(A)
$$


it follows that by taking $y$ as the equilihrium solution $x_{e}$ or any unnerturhed solution such as perlodic solution, if it exists, then it is stable. Note that $T_{t} x_{e}=x_{e}$. The above inequality holds for any $x, y \in D(A)$ which implies that a stability region is $D(A)$ and thus it can be extended to the whole space $H$ since $D(A)$ is dense in $H$ (cf. [9]). Therefore, the theorem is proved.

Theorem 3.2. Let A be the infinitesimal generator of a (1inear) negative contraction semi-group of class $C_{0}$ with contractive constant $B$. Assume that $f$ satisfies the condition ( 1 ) In theorem 3.1 and that

$$
(f(x)-f(y), x-y) \leqq k|| x-y||^{2} \text { with } k<\beta \text { for all } x, y \varepsilon \text { II. }
$$

Then all the results in theorem 3.1 hold. Moreover, if an equilibrium solution exists (or any unperturbed solution), it is exponentiallv asymptotically stable.

Proof. Let $A_{1}=A+f(\cdot)$. Since $A$ is the infinitesimal generator of a negative contraction semi-groun, it is densely defined, dissinative and $R(I-A)=H$. Applying theorem 2.1 for the linear case, A is strictly dissipative with dissipative constant $\beta$, that is

$$
(A x, x) \leqq-B|| x||^{2} \text { for a11 } x \in D(A) \text {. }
$$

Thus the onerator $A_{1}$ is strictly dissipative with dissinative constant $B-k$ since by the hyoothesis (3-2)

$$
\left(A_{1} x-A_{1} y, x-y\right)=(A x-A y, x-y)+(f(x)-f(y), x-y) \leqq-(B-k)|| x-y \|^{2}
$$

for all $x, y \in D\left(A_{1}\right)$. To show that $P\left(I-A_{1}\right)=H$, we prove $P\left(T-\alpha A_{1}\right)=H$ for some $\alpha>0$, since the monotonicity of $-\Lambda$ implies that $(T-\alpha A)^{-1}$ exists for every $\alpha>0$, and if $R(I-\alpha A)=H$ for some $\alpha>0$ then $R(I-A)=H$ (cf. [5]). The reason for doing this is that if the same argument as in the proof of theorem 3.1 is used 1 t will lead to the unnecessary 
requirement $k \leqq 1$. Let $I-\alpha A_{1}=-\alpha A+(I-\alpha f(\cdot))=G+G_{0}$ where $G=-\alpha A_{\text {, }}$ and $G_{0}=I-\alpha f(\cdot)$. Since $A$ is the Infinitesimal generator of a semi-groun, $\alpha \varepsilon \rho(A)$ (the resolvent set of A) for all $\alpha>0$ (cf. [11]) which implies that $R(I+C)=R(I-\alpha A)=H$. The mapping $G_{0}=I-\alpha f(\cdot)$ is monotone for $\alpha \leqq \mathrm{k}^{-1}$ since by the assumption (ii) $\left(G_{0} x-G_{0} y, x-y\right)=(x-y, x-y)-\alpha(f(x)-f(y), x-y) \geqq(1-\alpha k)|| x-y \|^{2} \geqq n$. It is obvious by the assumption (i) that $G_{0}$ is continuous on $H$ in the strong topology to the weak topology and is bounded on every hounded subset of $H$. Finally, the relation ||$G x+G_{0} x|| \rightarrow \infty$ as $\|x\| \rightarrow \infty$ is also satisfied. This is due to the fact that the dissipativity of $x \mathrm{~A}$ and the relation (3-1) imply that

$$
\| G x+G_{0} x|| \geqq\left(-\alpha A x+G_{0} x, x\right) /|| x|| \geqq((x, x)-\alpha(f(x), x)) /|| x|| \geqq|| x||-x|| f(0)||
$$

where $\alpha>0$ is a fixed number. Hence by choosing $\alpha \leqq k^{-1}$, all the hypotheses in theorem 5 of [2] are satisfled and the result $R(I-x A)=R\left(C_{S}+G_{0}\right)=H$ follows. It should be noted that $k>0$ so that $n<\alpha \leq k^{-1}$ exists. (if $\mathrm{k} \leqq 0$, then $\mathrm{G}_{0}$ is monotone by taking, for instance, $\alpha=1$ and the other conditions remain unchanged). By theorem 2.1, $A_{1}$ is the infinitesimal generator of a nonlinear negative contraction semi-groun $\left\{\mathrm{T}_{t} ; t \geq 0\right\}$ on $D(A)$ with the contractive constant $\beta-k$. Therefore the results in the theorem follow directly from the negative contraction pronerty of the semi-groun $\left\{T_{t} ; t \geqq 0\right\}$.

Remark. If A ts the infinitesimal generator of a contraction semi-groun instead of a negative contraction semi-group, any unperturbed solution is st111 asymptotically stable provided that the constant $k$ appearing in the condition (3-2) is negative, since in this case, we mav take $B=0$ and the operator $A_{1}=A+f(\cdot)$ remains strictly dissipative with dissipative constant $-k$. The proof of $R(I-A)=H$ remains the same. 
Corollary 1. Under the hynotheses of theorem 3.1 (theorem 3.2) and in addition, if $f(n)=n$, then the null solution is stahle (asymptotically stable) with the stability region the whole space $\mathrm{P}$.

Proof. If $f(0)=0$ then $x(t) \equiv 0$ is an equilibrium solution (called the null solution) of (1-1). Hence by theorem 3.1 (resp., theorem 3.2), the null solution is stable (resp., asymptotically stable) with the stability region extended to the whole space $\mathrm{H}$.

Corollary 2. Let A be the Infinitesimal generator of a (Iinear) negative contraction semi-group of class $C_{0}$ with contractive constant $B$, and let $f$ be Lipschitz continuous on $H$ with Lipschitz constant $k<B$, that is

$$
\|f(x)-f(y)\| \leqq k|| x-y|| \text { for all } x, y \varepsilon H \text {. }
$$

Then for any $x \in D(A)$ there exists a unique solution $T_{t} x$ to (1-1) with $T_{0} x=x$ such that any equilibrium solution $x_{e}$ to $(1-1)$ is asymptotically stahle. In particular, if $f(n)=n$ the null solution is asymptoticallv stable. Moreover, a stability region is $D(A)$ which can be extended to the whole space $H$.

Proof. By the Lipschitz continuity of $f$ on $H$, it follows that condition (i) In theorem 3.1 is satisfied. This is due to the fact that strong continuity implies weak continuity, and by (3-3) with $x_{0}$ a fixed - lement in $\mathrm{H}$

$$
\|f(x)\| \leqq\left\|f\left(x_{0}\right)\right\|+k|| x||+k|| x_{0} \|
$$

which is bounded whenever ||$x||$ is bounded. Moreover, by (3-3)

$$
(f(x)-f(y), x-y) \leqq\|f(x)-f(y)\||| x-y\|\leqq k|| x-y\|^{2}
$$

and so condition (3-2) in theorem 3.2 is satisfied. llence, by theorem 3.2 the existence and the uniqueness of a solution as well as the stability property of an equilibrium solution are proved. In particular, if $f(0)=0$ then corollary 1 implies that the null solution is asymptoticallv stable. 
It is obvious that under the hypotheses of theorem 3.2.

and in addition if an equilibrium solution $x_{e}$ exists then it is uninue since if $y_{e}$ is another enilihrfum solution, the neqative contraction nronerty of any two solutions to (1-1) Imnlies that

$$
|| x_{e} e^{-v} e \| \leqq e^{-(\beta-k) t}|| x_{e} e^{-v_{e}}|| \quad \text { for all } t \geqq 0
$$

which is imnossible unless $x_{e}=y_{e}$. Note that $T_{t} x_{e}=x_{e}$ and $T_{t}{ }^{y}=v_{e}$ for all $t \geqq 0$. The following, theorem gives weaker conditions on $A_{0}$ and on $f$ for the uniqueness of an equilibrium solution. Theorem 3.3. Let the linear onerator $A$ annearing in (1-1) be such that $\cap \in D(A)$ and that for some fintte number $R(1 . e .,|R|<\infty)$,

$$
(A x, x) \leqq P(x, x) \quad \text { for all } x \in D(A) \text {. }
$$

Let $f$ he defined on $D(A)$ to $Y$ such that $f(n)=n$ and such that for some finte number $k$ (f.e., $|k|<\infty)$

$$
(f(x), x) \leqq k\|x\|^{2} \quad \text { for al1 } x \in \Gamma(A) \text {. }
$$

If $\beta>k$ then the null solution of $(1-1)$ is the onlv eanilihrium solution.

proof. Tt is obvious that the zero vector is an enuflibrium solution of $(1-1)$. Let $x_{e}$ he any other equilibrium solution, then $x_{e} \varepsilon D(A)$ and by the definition of an enuilibrium solution, $A x_{e}+f\left(x_{e}\right)=n$. It follows that

$$
0=\left(A x_{e}+f\left(x_{e}\right), x_{e}\right)=\left(A x_{e}, x_{e}\right)+\left(f\left(x_{e}\right), x_{e}\right) \leqq-(B-k)|| x_{e} \|^{2}
$$

which imnlies that $x_{e}=0$ since by hynothesis $B-k>0$. Iience the uninueness of the equilibrium solution is proved.

Most of the theorems developed in this section un to now assumed that the 11near part A of $(1-1)$ is the infinitesimal generator of a contraction semi-groun of class $r_{0} \cdot$ A necessary and sufficient condition for A having this pronerty is that $A$ is dissinative, $D(A)$ is dense in $I$ 
and $\mathrm{D}(I-A)=\mathrm{II}$ (cf. [7]). Ngain the reaufrement $R(I-A)=H$ means the existence of a solution of the functional equation

$$
\mathbf{x}-\mathbf{A x}=\mathbf{z}
$$

for every $z \in I I$ which hy itself needs further fustification. Torever in case $A$ is a self-afoint onerator which occurs of ten in nhystcal annlications, this requirement can he eliminated in these theorems. In order to show this, we annly a theorem from [1] due to Brotder by considertng a denselv defined closed onerator and then take a selfadjoint onerator as a specfal case.

Theorem 3.4. Jet A he a densely defined closed onerator from $H$ into $H$. Sunnose that: (1) A is strictly dissipative with dissinative constant $R,(i i) A^{*}$ is the closure of $i$ ts restriction to $D(A) \cap D\left(A^{*}\right)$ where $A^{*}$ is the adjoint operator of $A$, (iii) $f$ satisfies the conditions (1) and (3-2) in theorem 3.2. Then all the results in theorem $3 . ?$ hold. Proof. Let $A_{1}=A+f(\cdot)$, then $A_{1}$ is strictly dissinative, since by hvoothesis

$$
\left(A_{1} x-A_{1} y, x-y\right)=(A x-A v, x-v)+(f(x)-f(v), x-y) \leqq-(B-k)|| x-v \|\left.\right|^{2}
$$

for all $x, y \in D(A)=D\left(A_{1}\right)$. To show that $R\left(I-A_{1}\right)=H$, let $T=I-A_{1}=-A+(I-f(\cdot))$, then $D(T)=D(A)$ is densely defined. Since $-A$ is densely defined, $A$ * exists and ts closed, and by the assumption (i1) $-A^{*}$ is the closure of its restriction to $D(-A) \cap D\left(-A^{*}\right)$. By (iii) the operator $G=I-f(\cdot)$ is continuous from all of $\mathrm{H}$ to $\mathrm{H}$ in the strong topology to the weak tonology which implies its hemi-continuity from $H$ to $H$ with $D(C)=H$. The houndedness of $G$ on hounded subsets of $H$ also follows from (11i). Moreover

$$
(\mathrm{Tx}-\mathrm{Ty}, \mathrm{x}-\mathrm{v})=(\mathrm{x}-\mathrm{y}, \mathrm{x}-\mathrm{y})-\left(\mathrm{A}_{1} \mathrm{x}-\mathrm{A}_{1} \mathrm{y}, \mathrm{x}-\mathrm{y}\right) \geq(1+\beta-k)|| x-y \|^{2} \quad \mathrm{x}, \mathrm{y} \varepsilon \mathrm{n}(\mathrm{T})
$$

so that $T$ is monotone. In particular by letting $y=n(n \varepsilon n(A)=\Gamma(T))$ in the above inenuality and since $T \cdot n=n-A_{1} \cdot n=-f(0)$, it follows that 


$$
\begin{gathered}
(T x, x) \geqq(1+\beta-k)|| x||^{2}-(f(0), x) \geqq((1+B-k)|| x||-|| f(n)||)|| x|| \\
\quad \text { for all } x \in D(T),
\end{gathered}
$$

and since $B-k>0$ the real valued function $c(|| x||)$ defined hv

$$
c(|| x \|)=(1+B-k)|| x||-\|f(0)\|
$$

has the nronerty that $c(|| x||) \rightarrow \infty$ as $\|x\| \rightarrow \infty$. Wence a11 the condtions in theorem 1 of [1] are satisfied if we take, for instance, the completelv continuous manping $C=0$ (the zero operator which mans all $x \in H$ into the $n$ vector in $H)$. Therefore $R\left(I-A_{1}\right)=R(T)=H$. By annlving theorem 2.1, $A_{1}$ is the infinitesimal generator of a non-linear negative contraction semi-group on $D\left(A_{1}\right)=D(A)$ with the contractive constant $R-k$. Thus, the stated results in the thoerem follow directly from the negative contraction semi-groun property as in the nroof of theorem 3.1 .

Remark. The above theorem can also be proved with $\beta=k=n$, in which case the equilibrium solution is stable with a stability region $D(A)$. The proof is exactly the same by letting $B=k=0$.

Since an unbounded self-adjoint operator $A$ is a denselv defined closed onerator having the property that $D(A)=D\left(A^{*}\right)$ (in fact $A=A^{*}$ ) we have, with a stronger assumption on the function $f$, the following result which is stated as a theorem becasue of its usefulness in anplications. Theorem 3.5. Let $A$ be an unbounded self-adjoint operator from $\eta$ to $H$ and assume that it is strictly dissipative with dissinative constant $\beta$. Let $f$ be Iipschitz continuous on $H$ with Linschitz constant $k<B$, that is

$$
|| f(x)-f(y)|| \leqq k|| x-v|| \quad \text { for a11 } x, y \in H \text {. }
$$

Then all the results in theorem 3.2 hold. 
Proof. The self-adjointness of $A$ implies that $A$ is a denselv defined closed onerator and $n\left(A^{*}\right)=D(A)$ (in fact, $A^{*} A^{*}$ ). Thus condition (ii) in theorem 3.4 is satisfied. By the Lipschitz continuity of $f, f$ is continuous in the strong tonology and is hounded on every bounded subset of $H$. This assumption (Lipschitz contfnuitv) also imnlies that

$$
(f(x)-f(y), x-y) \leqq\|f(x)-f(y)\||| x-y\|\leqq k|| x-v\|^{2} \text { for all } x, y \varepsilon \text {. }
$$
Ience, all the conditions in theorem 3.4 are satisfied, and the results follow hy annlving that theorem.

Remark. The Linschitz continuity of $f$ in the theorem can be weakened by using the conditions (i) and (3-2) in theorem 3.2 .

It is easily seen from theorems 2.1 and 2.2 that stability and asymptotic stability are invartant if the inner product $(\cdot, \cdot)$ of $1 \mathrm{t}$ is renlaced by an equivalent inner product $(,,)_{1}$ with respect to which $A_{1}=A+f(\cdot)$ is dissipative. Recause of its usefulness in applications (for instance, a non-self-adjoint operator in a Ililbert snace $(H,(\cdot, \cdot)$ ) can sometimes he made self-adjoint in $\left(\pi,(\cdot, \cdot)_{1}\right)$ where $(\cdot, \cdot)_{1}$ is an equivalent inner product) we show the following theorem.

Theorem 3.6. Let A he a densely defined linear operator from $H=(H,(\cdot, \cdot))$ into $H$, and let $f$ satisfy the condition (i) in theorem 3.1 . If there exists an equivalent inner product $(\cdot, \cdot)_{1}$ such that $A$ is a self-adjoint onerator in $H_{1}=\left(H,(\cdot, \cdot)_{1}\right)$ satisfying.

$$
(A x, x)_{1} \leqq-B|| x||_{1}^{2} \quad x \in D(A)
$$

and if

$$
(f(x)-f(y), x-y)_{1} \leqq k|| x-y \|_{1}^{2} \text { with } k<\beta, \quad x, y \varepsilon I .
$$

Then, all the results stated in theorem 3.4 are valld.

Proof. Consider A as an onerator from the space $\mathrm{H}_{1}=\left(H,(\cdot, \cdot)_{1}\right)$ into $H_{1}$. Since $A$ is self-adjoint in the space $H_{1}$, it is a denselv defined 
closed onerator and $D(A)=D\left(A^{*}\right)$. The continutty and the boundedness of $f$ with resnect to the $\|\cdot\|$-norm topology implies the same property of $f$ with respect to the $\left.\|\cdot\|\right|_{1}$-norm topology since these two norms are equivalent. By assumption, A is strictly dissipative and the condition (iii) in theorem 3.4 is satisfied with resnect to $(\cdot, \cdot)_{1}$. Hence all the hypotheses in theorem 3.4 are satisfied by considering $\mathrm{H}_{1}$ as the underlying space which implies that the operator $A_{1}=A+f(\cdot)$ is the infinftesimal generator of a nonlinear negative contraction semi-groun $\left\{T_{t} ; t \geqq 0\right\}$ on $D(A)$ with contractive constant $B-k$ in the space $H_{I}$. $B y$ theorem 2.2, A is the infinitesimal generator of a nonlinear negative semi-group $\left\{T_{t} ; t \geq 0\right\}$ on $D(A)$, not necessartly contractive, in the original space $\mathrm{H}$. Therefore all the results in theorem 3.4 hold by the semi-groun properties.

4. Annlications to Partial Differential Equations

In this section, we shall give some applications of the results obtained in the previous section to a class of linear and semi-1inear partial differential equations which can serve as an illustration of some steps in apnlving the theorems develoned for operator differential equations. For simplicity, we limit our discussion to second order differential equations in an $\mathrm{n}$-dimensional Euclidian space $\mathrm{R}^{\mathrm{n}}$ and consider the Filhert space $L^{2}(\Omega)$ as the underlying space. In the following, the first simnle example of a linear differential equations gives a fairly detailed description of the application from which some more general equations can easily he obtained. Criteria for the existence and stability of solutions in terms of the coefficients of a given partial differential operator are stated as theorems which are concrete results of the application from the abstract operator differential equation. 
Example 4.1. Consider the 11near partial differential

equation

$$
\frac{\partial u}{\partial t}=a(x) \frac{\partial^{2} u}{\partial \chi^{2}}+b(x) \frac{\partial u}{\partial x}+c(x) u \quad x \in(n, 1)
$$

with the boundary conditions

$$
u(t, n)=u(t, 1)=n \quad(t \geqq n) .
$$

Assume that the coefficient $a(x)$ is positive on $[0,1]$ and that $a(x)$, $b(x), c(x)$ are all infinitely differentiable functions in an onen interval $I_{0}$ containing $[n, 1]$. Then the linear operator

$$
L=a(x) \frac{\partial^{2}}{\partial x^{2}}+b(x) \frac{\partial}{\partial x}+c(x)
$$

is an elliptic partial differential operator (cf. [4]). The formal adfoint onerator of $L$ is given as

$$
L *(\cdot)=\frac{\partial^{2}}{\partial x^{2}}(a(x)(\cdot))-\frac{\partial}{\partial x}(h(x)(\cdot))+c(x)(\cdot)
$$

which is also an elliptic partial differential operator. It is easily shown by a simple calculation that equation (4-1) can be reduced to the form

$$
\frac{\partial u}{\partial t}=\frac{1}{q(x)} \frac{\partial}{\partial x}\left(p(x) \frac{\partial}{\partial x}\right)+c(x) u
$$

where

$$
\begin{aligned}
& q(x)=(a(x))^{-1} e^{\int_{x}^{x}(b(\xi) / a(\xi)) d \xi}\left(x_{0} \in[n, x] \text { fixed }\right) \\
& p(x)=a(x) q(x) .
\end{aligned}
$$

Let us seek a solution in the real Hilbert space $L^{2}(0,1)$ in which the inner product between any pair of elements $u, v \varepsilon L^{2}(n, 1)$ is defined by

$$
(u, v)=\int_{0}^{1} u(x) v(x) d x
$$

Define the operator $T$ in $L^{2}(0,1)$ as the restriction of $L$ on $C^{\infty}(0,1)$ and $\hat{T}$ the restriction of $L^{*}$ on $C^{\infty}(0,1)$ by

$$
\begin{gathered}
D(T)=D(\hat{T})=\left\{u \in C^{\infty}([0,1]) ; u(0)=u(1)=0\right\} \\
T u=L u, \hat{T} u=L^{*} u \quad u \in D(T) .
\end{gathered}
$$


Let $A$ and $\hat{A}$ denote the closure of $T$ and $\hat{T}$ respectively $(T$ and $\hat{T}$ are closable). Then $D(A)$ is dense in $L^{2}(0,1)$ since $D(A)>D(T)>C_{0}^{\infty}(0,1)$ which is dense in $L^{2}(0,1)$. Thus $A^{*}$ and $(\hat{A}) *$ both exist. In general, $T$ is not self-adjoint with respect to the inner product defined in (4-5). However, by defining the scalar functional $(u, v)_{1}$ by

$$
(u, v)_{1}=(u, q v)=\int_{0}^{1} u(x) q(x) v(x) d x
$$

where the function $q(x)$ is the known function given in (4-4) it is easily seen that $(\cdot, \cdot)_{1}$ possesses all the propertles of an inner product. Since $(u, u)_{1}=(u, q u)=\int_{0}^{1} q u^{2} d x$, it follows that

$$
\left(\min _{0 \leqq x \leq 1} q(x)\right)|| u\left\|^{2} \leqq\right\| u\left\|_{1}^{2} \leqq\left(\max _{0 \leqq x \leqq 1} a(x)\right)|| u\right\|^{2}
$$

which implies that $(\cdot, \cdot)_{1}$ and $(\cdot, \cdot)$ are equivalent. Notice that $q(x)>0$ and is continuous over the closed interval $[0,1]$ so that it actual.1v attains its maximum and minimum values bounded away from zero and $\infty$. Moreover, for any $u, v \in D(T)$, on integrating by parts and taking notice that the boundary conditions are satisfied for any $u \in D(T)$ we have

$$
\begin{aligned}
& (u, T v)_{1}=(u, q T v)=\int_{0}^{1} u q\left[q^{-1} \frac{\partial}{\partial x}\left(p \frac{\partial v}{\partial x}\right)+c v\right] d x \\
& =\int_{0}^{1}\left[v \frac{\partial}{\partial x}\left(p \frac{\partial u}{\partial x}\right)+c q u v\right] d x=(T u, v)_{1}
\end{aligned}
$$

which shows that $\mathrm{T}=\hat{\mathrm{T}}$. It follows that (cf. Dunford and Schwartz [4] p. 1740) $A=(\hat{A}) *=A *$ which shows that $A$ is self-adjoint in the equivalent Hilbert space $L_{1}^{2}(0,1)$ equipped with the Inner product $(\cdot, \cdot){ }_{1}$. Moreover, the above equality implies that for any $u \in D(T)$

$$
(u, T u)_{1}=-\int_{0}^{1}\left[p\left(\frac{\partial u}{\partial x}\right)^{2}-c q u^{2}\right] d x=-\int_{0}^{1}\left[a \quad a\left(\frac{\partial u}{\partial x}\right)^{2}-c a u^{2}\right] d x \text {. }
$$

On setting $u_{1}=a^{1 / 2} u$ then ||$u_{1}||=|| u||_{1}$ and by an elementary calculation we have

$$
a q\left(\frac{\partial u}{\partial x}\right)^{2}=a\left(\frac{\partial u_{1}}{\partial x}\right)^{2}-\frac{1}{2}\left(b-a^{\prime}\right) \frac{\partial u_{1}^{2}}{\partial x}+\frac{1}{4} \frac{\left(b-a^{\prime}\right)^{2}}{a} u_{1}^{2}
$$


where $a^{\prime} \equiv \frac{d}{d x} a(x)$. Hence, integrating by parts and using, the well known inequality

$$
\int_{0}^{1}\left(\frac{d u}{d x}\right)^{2} d x \geq \pi^{2} \int_{0}^{1} u^{2} d x
$$

which is valid for any $u(x)$ satisfying the condition (4-2), we have

$$
\begin{aligned}
& (u, T u)_{1}=-\int_{0}^{1}\left[a\left(\frac{\partial u_{1}}{\partial x}\right)^{2}+\left(\frac{1}{2}\left(b^{\prime}-a^{\prime \prime}\right)+\frac{1}{4} \frac{\left(b-a^{\prime}\right)^{2}}{a}-c\right) u_{1}^{2}\right] d x \\
& \leqq-\int_{0}^{1}\left[\pi^{2} a_{m i n}+\frac{1}{2}\left(b^{\prime}-a^{\prime \prime}\right)+\frac{1}{4} \frac{\left(b-a^{\prime}\right)^{2}}{a}-c\right] u_{1}^{2} d x \leqq-\left.\beta|| u\right|_{1} ^{2}
\end{aligned}
$$

where

$$
\begin{gathered}
a_{\min }={ }_{n \leqq x \leqq 1}^{\min } a(x) \\
\beta=\min _{0 \leqq x \leqq 1}\left[\pi^{2} a_{\min }+\frac{1}{2}\left(b^{\prime}(x)-a^{\prime \prime}(x)\right)+\frac{1}{4} \frac{\left(b(x)-a^{\prime}(x)\right)^{2}}{a(x)}-c(x)\right] \cdot(4-10)
\end{gathered}
$$

It follows that if $\beta=0$ or $\beta>0$ then $T$ is dissipative or strictly dissipative, respectively, with respect to $(\cdot, \cdot)_{1}$. The dissinativity or strict dissipativity of $\mathrm{T}$ implies the dissipativity or strict dissipativity, respectively, of $A$. To see this, let $u \in D(A)$ then by the definition of the closure of a closable operator there exists a sequence $\left\{u_{n}\right\} \in D(T)$ such that $u_{n} \rightarrow u$ and $\lim _{n \rightarrow \infty} T u_{n}$ exists and equals $A u$ (cf. [11]). Hence by the continuity of inner product, we have

$$
(A u, u)_{1}=\lim _{n \rightarrow \infty}\left(T u_{n}, u_{n}\right)_{1} \leqq \lim _{n \rightarrow \infty}\left(-B|| u_{n} \| l_{1}^{2}\right)=-\left.B|| u\right|_{1} ^{2}
$$

which shows the dissipativity and strict dissinativity of A. Therefore, by applying theorems 3.6 and 3.3 with $f \equiv 0$ we have the following results. Theorem 4.1. Assume that the coefficients $a(x), b(x)$ and $c(x)$ of 4-1 are infinitely differentiable over any open interval $I_{0}$ containing $[0,1]$ and that $a(x)$ is positive on $[0,1]$. If the condition $(4-10)$ is satisfied, then for any initial element $u_{0}(x) \in D(A)$ there extsts a unique solution $u(t, x)$ in the sense of definition 1.1 with $u(0, x)=u_{0}(x)$. Moreover, 
the null solution of $(4-1)$ is stable if $\beta=0$ and is asymptotically stable if $\beta>0$ and in the later case the null solution is the only equilibrium solution.

As an example of the above theorem, take $a(x)=\frac{1}{R}, b(x)=\frac{2}{\sqrt{R}} x$, $c(x)=\left(x^{2}+\frac{2}{\sqrt{R}}\right)$ where $R$ is a positive constant to be determined, then

$$
B=\min _{0 \leqq x \leqq 1}\left[\frac{\pi^{2}}{R}+\frac{1}{\sqrt{R}}+\frac{1}{4} R\left(\frac{2}{\sqrt{R}} x\right)^{2}-\left(x^{2}+\frac{2}{\sqrt{R}}\right)\right]=\frac{\pi^{2}}{R}-\frac{1}{\sqrt{R}} .
$$

Hence $B>0$ if $0<R<\pi^{4}$ which shows the same result as given in [3].

Remark. The solution $u(t, x)$ in theorem 4.1 is in fact a

solution of (4-1) in the strong sense $i . e ., \frac{d u(t, x)}{d t}=A u(t, x)$ in the norm topology (cf. [7]). However, in the case of semi-1inear equations, it is not certain that this is the case. Thus, we shall assume that any solution in the following discussion is in the sense of definition 1.1 .

Example 4.2. Consider the partial differential equation

$$
\frac{\partial u}{\partial t}=a(x) \frac{\partial^{2} u}{\partial \chi^{2}}+b(x) \frac{\partial u}{\partial x}+c(x) u+f(x, u)
$$

with the boundary conditions $u(t, 0)=u(t, 1)=0$ where $a(x), b(x), c(x)$ are the same as in theorem 4.1 and $f$ is a nonlinear function defined on $\mathrm{L}^{2}(0,1)$ to $\mathrm{L}^{2}(0,1)$. According to theorem 3,6 , if $f$ is continuous on $L^{2}(0,1)$ and 1 s bounded on bounded subsets of $L^{2}(0,1)$ such that

$$
(f(x, u)-f(x, v), u-v)_{1} \leq k_{1}|| u-v \|_{1}^{2} \text { with } k_{1}<\beta, u, v \varepsilon L^{2}(0,1)
$$

where $(., .)_{1}$ is the equivalent inner product defined $\ln (4-6)$ and $\beta$ is given by (4-10), then all the results in theorem 4.1 with respect to an equilibrium solution, if it exists, remain valid. In particular if $f(0)=0$, the null solution is exponentially asymptotically stable.

To 1llustrate the above statement take, for example, the function

$$
f(x, u)=k(x) \frac{u^{2}}{\lambda^{2}+u^{2}} \quad\left(\lambda^{2}>0\right) \text {. }
$$


where $k(x)$ is a bounded function on $[0,1]$. It is obvious that $f$ is continuous on $L^{2}(n, 1)$ (in the strong topology) and is hounded on $L^{2}(0,1)$. By the definition of $(., .)_{1}$ in $(4-6)$

$$
\begin{gathered}
(f(x, u)-f(x, v), u-v)_{1}=\int_{0}^{1} k(x)\left(\frac{u^{2}}{\lambda^{2}+u^{2}}-\frac{v^{2}}{\lambda^{2}+v^{2}}\right) a(u-v) d x \\
=\lambda^{2} \int_{0}^{1} \frac{k(x)(u+v)}{\left(\lambda^{2}+u^{2}\right)\left(\lambda^{2}+v^{2}\right)} q(u-v)^{2} d x \leqq \\
\leqq \lambda^{2} \max _{0 \leqq x \leqq 1}\left[\frac{|k(x)(u(x)+v(x))|}{\left(\lambda^{2}+u^{2}(x)\right)\left(\lambda^{2}+v^{2}(x)\right)}\right]|| u-\left.v\right|_{1} ^{2} .
\end{gathered}
$$

It is easily shown that for any real number $u, v$

$$
\frac{|u+v|}{\left(\lambda^{2}+u^{2}\right)\left(\lambda^{2}+v^{2}\right)}<\frac{1}{\left|\lambda^{3}\right|}
$$

which implies that

$$
(f(x, u)-f(x, v), u-v)_{1}<\left|\frac{k m}{\lambda}\right||| u-v \|_{1}^{2} .
$$

where $k_{m}=\max _{n \leq x \leqq 1} k(x)$ it follows that if $\left|\frac{k_{m}}{\lambda}\right| \leqq \beta$ then the existence and uniqueness of a solution for any initial element $u_{0}(X) \in D(A)$ are ensured. Moreover the null solution is exponentially asymptotically stahle with stabilitv region $D(A)$.

Example 4.3. Consider the second order linear differential equations of the form

$$
\frac{\partial u}{\partial t}=\sum_{i, j=1}^{n} \frac{\partial}{\partial x_{i}}\left(a_{1 j}(x) \frac{\partial u}{\partial x_{j}}\right)+c(x) u \quad x \in \Omega
$$

with the boundary conditions

$$
u\left(t, x^{\prime}\right)=0 \quad x^{\prime} \varepsilon \partial \Omega \quad t \geqq 0
$$

where $x=\left(x_{1}, x_{2}, \ldots, x_{n}\right), \Omega$ is a bounded open subset of the Euclidean space $R^{n}$ with boundary $\partial \Omega$ which is a smooth surface and no noint in $\partial \Omega$ is interior to $\bar{\Omega}$, the closure of $\Omega$. Assume that $a_{i j}(x)=a_{j i}(x)$ $(1, j=1,2, \ldots, n)$ and together with $c(x)$ are Infinitely differentiable real-valued functions in a domain $\Omega_{0}$ which contains $\bar{\Omega}$ and that there 
exists a positive constant $x$ such that

$$
\sum_{i, j=1}^{n} a_{i j}(x) \xi_{i} \xi_{j} \geq \alpha \sum_{1=1}^{n} \xi_{1}^{2} \quad x \varepsilon \Omega_{0}, \xi \varepsilon R^{n} .
$$

The operator

$$
L=\sum_{i, j=1}^{n} \frac{\partial}{\partial x_{i}}\left(a_{i j}(x) \frac{\partial}{\partial x_{j}}\right)+c(x)
$$

is a strongly elliptic partial differential onerator in $\Omega_{\Omega}$.

It is easily seen by definition that the operator $L$ is

formally self-arjoint i.e., $L=L^{*}$. Let $T$ be the operator in $I^{2}(\Omega)$ defined by

$$
\left.\begin{array}{ll}
D(T)=\left\{u \in C^{\infty}(\bar{\Omega}) ;\right. & u\left(X^{\prime}\right)=0, X^{\prime} \varepsilon \partial \Omega
\end{array}\right\} \begin{array}{ll}
T u=L u & u \in D(T),
\end{array}
$$

and let $A$ be the closure of $T$. By theorem 25 in [4] (p. 1743), A is self-adjoint. For any $u \in D(T)$, integration by parts yields

$$
\begin{aligned}
(u, T u) & =\int_{\Omega} u T u d x=\int_{\Omega}\left[\sum_{i, j=1}^{n} u \frac{\partial}{\partial x_{i}}\left(a_{i j}(x) \frac{\partial u}{\partial x_{j}}\right)+c(x) u^{2}\right] d x \\
& =-\int_{\Omega}\left[\sum_{i, j=1}^{n} a_{i j}(x) \frac{\partial u}{\partial x_{i}} \frac{\partial u}{\partial x_{j}}-c(x) u^{2}\right] d x
\end{aligned}
$$

where $d x=d x_{1} d x_{2} \ldots d x_{n}$. By the assumption (4-14) and using the well known inequality [12]

$$
\int_{\Omega} \sum_{i=1}^{n}\left(\frac{\partial u}{\partial x}\right)_{1}^{2} d x \geqq \gamma \int_{\Omega} u^{2} d x
$$

where $\gamma$ is a nositive real number, we obtain

$$
\begin{gathered}
(u, T u) \leqq-\int_{\Omega}\left[\alpha \sum_{i=1}^{n}\left(\frac{\partial u}{\partial x_{i}}\right)^{2}-c(x) u^{2}\right] d x \leqq-\int_{\Omega}(x \gamma-c(x)) u^{2} d x \\
\leqq-\left.\left(\alpha \gamma-c_{m}\right)|| u\right|^{2}=-\left.\beta|| u\right|^{2}
\end{gathered}
$$

where $c_{m}=\max _{\chi \in \bar{\Omega}} c(x)$ and $\beta=\alpha \gamma-c_{m}$. Hence, $T$ is dissipative if $\beta=0$ and is strictly dissipative if $\beta>0$. The disslpativity and strict 
dissinativity of $\Lambda$ follow from the dissipativity and strfct dissipativity, resnectively, of $T$ as has been shown in example 4.1 since $A$ is the closure of $T$. Therefore, A satisfies all the hypotheses in theorem 3.5. To summarize, we have:

Theorem 4.2. Assume that $a 11$ the real-valued functions $a_{i j}(x)=a_{j 1}(x)$ $(1, j=1,2, \ldots, n)$ and $c(x)$ in enuation $(4-12)$ are infinitely differentiable in a domain $\Omega_{0}$ containing $\bar{\Omega}$, the closure of $\Omega$, where $\Omega$ is a bounded open set in $R^{n}$ whose boundary $\partial \Omega$ is a smooth surface and no noint of $\partial \Omega$ is interior to $\bar{\Omega}$. If the condition (4-14) is satisfied and if

$$
\beta=\alpha \gamma-\frac{\max }{x \varepsilon \bar{\Omega}} c(x) \geqq 0
$$

where $\alpha$ is given in $(4-14)$ and $\gamma$ is given in (4-15), then for any $u_{o}(x) \in D(A)$ there exists a unique solution $u(t, x)$ to (4-12) strongly continuous in $t$ with respect to the $\mathrm{L}^{2}(\Omega)$ norm with $\mathrm{u}(n, \mathrm{x})=\mathrm{u}_{0}(\mathrm{x})$. Moreover, the null solution is stable for $\beta=0$ and is asymptotically stable if $B>n$ and in the later case the null solution is the only enuilibrium solution. The stability region is $D(\Lambda)$ which can be extended to the whole snace $\mathrm{L}_{2}(\Omega)$.

It is seen from the above theorem that the major conditions imposed on the coefficients of the operator $L$ are conditions (4-14) and (4-16). Notice that if $c(x)$ is a non-positive function, then (4-16) is automaticallv satisfied. As a special form of (4-12) we consider the equation

$$
\frac{\partial u}{\partial t}=\sum_{i=1}^{n} \frac{\partial}{\partial x_{1}}\left(a_{i}(x) \frac{\partial u}{\partial x_{1}}\right)+c(x) u \quad x \varepsilon \Omega
$$

with the boundary conditions (4-13). The following result is an immediate consequence of theorem 4.2 .

Corollary. Assume that the real-valued functions $a_{i}(x)(1=1,2, \ldots, n)$ and $c(x)$ in equation (4-17) are infinitely differentiable in a domain $\Omega_{0}$ 
containing $\bar{\Omega}$ where $\Omega$ is a bounded open set in $\mathrm{R}^{\mathrm{n}}$ whose houndary $\partial \Omega$ is suffictently smooth. If, in addition, $a_{1}(x)$ is positive for each 1 and $c(x)$ is non-positive then all the results in theorem 4.2 hold.

Proof. Consider $(4-17)$ as a special form of (4-12) with $a_{i j}(x)=a_{i}(x)$ for $i=1$ and $a_{i j}(x)=n$ for $1 \neq j$. Then the condition (4-14) is satisfied since by hypothesis $\alpha=\min _{1 \leq 1 \leq n}\left(\min _{x \in \bar{\Omega}} a_{1}(x)\right)>0$ which implies

$$
\sum_{i, j=1}^{n} a_{i j}(x) \xi_{i} \xi_{j}=\sum_{i=1}^{n} a_{i}(x) \xi_{i}^{2} \geq \alpha \sum_{i=1}^{n} \xi_{i}^{2} .
$$

The condition (4-16) follows from the non-nositivity of $c(x)$. Hence the results follow by apnlving theorem 4.2 .

As an example of the above theorem, constder the equation

$$
\left.\frac{d u}{d t}=\Delta u-c^{2} u \quad \text { (c rea } 1\right)
$$

where $\Delta$ is the Lanlacian operator in $\Omega \in R^{3}$ with $\partial \Omega$ sufficiently smooth. Then all the conditions in the above theorem are fulfilled since in this case $a_{i}(x)=1$ for each 1 and $c(x)=-c^{2}$.

Just as one-dimensional space case, semi-linear equations of the form

$$
\frac{\partial u}{\partial t}=\sum_{i, j=1}^{n} \frac{\partial}{\partial x_{i}}\left(a_{i j}(x) \frac{\partial u}{\partial x}\right)+c(x) u+f(x, u) \quad x \varepsilon \Omega
$$

with the boundary conditions

$$
u\left(t, X^{\prime}\right)=0 \quad x^{\prime} \varepsilon \partial \Omega
$$

can similarly be treated where $f$ is a function on $\mathrm{L}^{2}(\Omega)$ to $\mathrm{L}^{2}(\Omega)$. For the sake of application, we state a theorem which is the consenuence of theorem 3.5 .

Theorem 4.3. Suppose that the semi-linear equation (4-18) with the boundary conditions (4-19) possesses the same linear part as given in theorem 4.2. If $f$ satisfies the conditions (i) and (3-2) in theorems 3.1 and 3.2 , respectively, where $B$ is given by $(4-16)$. Then (a) For any 
$u_{0}(x) \in D(\Lambda)$ there exists a unique solution of $(4-18)$ with $u(n, x)=u_{0}(x)$.

(b) An equilihrium solution (or a perfodic solution), if it exists,

is stable if $k=\beta$; and is asymptotically stable if $k<\beta$. (c) A stability region of the equilihrium solution is $D(A)$ which can be extended to the whole snace $L^{2}(\Omega)$.

References

[1] Browder, F. F. Nonlinear Tquations of Evolution. Ann, of Math., 80 (1964), 485-523.

[2] Browder, F. E. Nonlinear Equations of Fvolution and Nonlinear Accretive Operators in Banach Spaces. Bull. Amer. Math Soc., 73 (1967), 867-874.

[3] Buis, G. R. Jyapunov Stabllity for Partial Differential Eauations. Part I, NASA CR-1100, 1968.

[4] Dunford, N. and Schwartz, J. Linear Onerators. Vo1. 1, Nev York: Interscience, 1963.

[5] Kato, T. Nonlinear Semi-groups and Evolution Equations. J. Math. Soc. Japan, 19 (1067) 508-520.

[6] Kōmura, Y. Nonlinear Semi-grouns in Hilbert Space. J. Math. Soc. Jaban, 19 (1967), 403-5n7.

[7] Lumer, G. and Phillips, R. S. Dissipative Operators in a Banach Snace. Pactfic J. Math., 11 (1961), 679-698.

[8] Minty, G. J. Monotone (nonlinear) Operators in Hilbert Space. Duke Math. J., 29 (1962) 341-346.

[9] Pao, C. V. The Existence and Stability of Solutions to Nonlinear Operator Differential Equations (to appear).

[10] Vogt, W. G., Eisen, M. M. and Buis, G. R. Contraction Groups and Equivalent Norms. Nagoya Math. J., Vol. 34 (to appear), also Lyapunov Stability for Partial Differential Equations. Part II, NASA CR-1100, 1968.

[11] Yosida, K. Functional Analysis. Ber11n: Springer-Verlag, 1966.

[12] Zubov, V. I. Methods of A. M. Lyapunov and Their Application.

The Netherlands: P. Noordhoff Ltd., 1964. 